International Journal of Automotive and Mechanical Engineering ISSN: 2229-8649 (Print); ISSN: 2180-1606 (Online)

Volume 15, Issue 1 pp. 4919-4934 March 2018

(C) Universiti Malaysia Pahang, Malaysia

DOI: https://doi.org/10.15282/ijame.15.1.2018.4.0383

\title{
Improvement in Steering Performance by Push-Pull Operation in Car Driving
}

\author{
S. Kajiwara \\ Faculty of Science and Engineering, Kindai University, \\ 3-4-1 Kowakae Higashiosaka, Osaka, Japan \\ Email: kajiwara@mech.kindai.ac.jp \\ Phone: +81643073485 ; Fax: +81643073485
}

\begin{abstract}
Collision avoidance is one of the most difficult driving operations in the domain of intelligent vehicles. Steering operation error is one of the causes of traffic accidents. Steering operability also contributes greatly to the comfort of drivers and their mental workload during driving. Thus, with comfortable steering operation it is possible to reduce shoulder stiffness and arm fatigue caused by long-time driving. The development of automatic driving vehicles is becoming advanced worldwide. Consequently, the goal is to implement a level 3 automatic driving vehicle for practical use, in which "the system performs the acceleration, the steering, and the braking, and the driver responds when the system requests it". For this system to be practical, when autonomous driving becomes impossible for some reason, it is important to develop a system that allows the driver to be in control regardless of driving experience or age. In conventional round steering wheel, since there is no output muscle directly involved in the direction of movement of the upper limbs, it is difficult to instantaneously exert a large force and control performance is not good. Therefore, a twin lever steering (TLS), which is a push / pull alternate steering system, is proposed in this study. In the proposed TLS, there are output muscles that are directly involved in the direction of movement of the upper limb, including the biceps brachii muscle, and it is conceivable that the motor controllability is higher than in the conventional system. In this research, the superiority of the proposed TLS was verified using an experimental racing kart and driving simulator (DS). Furthermore, using DS, the superiority of a TLS in the steering operation immediately after the cancellation of automatic operation is clear.
\end{abstract}

Keywords: Autonomous car; lever steering; vehicle control; reaction time; mental workload; collision avoidance.

\section{INTRODUCTION}

Mistakes in steering operation are cited as one cause of traffic accidents. Steering operability contributes greatly to the comfort of the driver when driving a car and with a comfortable steering operation $[1,2]$; it is possible to reduce shoulder stiffness and arm fatigue caused by long-term driving [3]. Various types of assistances devices were designed to assist lateral control. A classification of those devices based on the combination of two human-automation interaction frameworks is proposed [4]. The development of automatic driving vehicles has advanced worldwide. Level 3 automatic driving vehicles are being put into practical use, in which automatic operation is said to be "a state where all of the acceleration, steering, and braking operations are performed by the system and the system is requested by the driver". In the future, to practically 
implement a level 3 automatic driving system, in the event that automatic driving becomes impossible for some reason, and the system is required to become redundant, the development of a system that the driver can control regardless of driving experience and age becomes important. Considering this, a steering operation system that has high motion controllability and enables a space-saving layout compared with the conventional steering wheel, such as a twin lever steering (TLS) [5] is useful. Upper limb movement can be considered motion in a two-dimensional plane composed of a shoulder joint, an elbow joint, and a carpal joint. In the case of round steering, the steering operation is performed by vertical movement. For this reason, a strong force is required to maintain the constant steering angle of turn during cornering. On the other hand, since the TLS operates the steering with a normal (forward and backward) motion, the steering angle can be maintained with a weaker force. In some cases, the body movement during steering operation was analyzed using a musculoskeletal model. Regarding the musculoskeletal model of the upper limb, it has also been reported that it is modeled as a three-dimensional seven-degree of freedom system linked to the shoulder [6]. A driver's muscle activation strategy during driving was validated using a mathematical model by measuring the driver muscle activity using electromyography (EMG) [7]. It was revealed that hand rigidity depends on the posture of the arm by measuring the hand-tip displacement and the handend force immediately after applying the forced displacement [8].

In normal round steering, it has been reported that the standard deviation of the arm positioning error when the elbow flexion angle is $\pi / 2$ is about $0.07 \mathrm{rad}$ [9]. This error is $0.11 \mathrm{rad}$ when converted to a steering angle with an elbow length of $0.3 \mathrm{~m}$ and a steering wheel radius of $0.19 \mathrm{~m}$. That is difficult to control since the initial response of steering straight ahead falls within the range of $0.11 \mathrm{rad}[10]$. Therefore, in order to steer with an accuracy less than this error, a steering reaction force is necessary. However, the control resolution is considered to be high in the longitudinal direction. Furthermore, to study the redundancy of automatic driving vehicles, we examine the safety and usefulness of a TLS and ordinary round type steering. A number of level 2 automatic driving vehicles are in practical use and the practical application of level 3 vehicles is currently being studied. Automatic driving level 3 is a state in which the driver and the automatic driving system coexist to perform the driving operation. Resuming manual control is not only a problem of action performance but is also related to the reorganization of drivers' visual strategies linked to drivers' disengagement from the steering task. Assistance designers should pay attention to potential changes in drivers' activity when carrying out development work on highly automated vehicles [11]. Automation promotes extended eyes-off-road behavior that may lead to a loss of situational awareness degrading a driver's ability to detect hazards and make necessary overrides [12]. In order to cope with level 3 automatic driving, it is necessary to develop a system that can safely change the driving control to the driver in an emergency situation where human judgment is necessary.

In other words, it is necessary to consider the steering operation performance immediately after the automatic operation is canceled. Even with manual operation, the reaction time is extended when conducting a selection reaction instructing different operation directions by stimulation. The operations carried out by the driver at the time of driving are accelerator, brake, and left and right turning operations. It is estimated that the reaction time for this driving behavior is longer than in the case of the simple reaction because the driver must select one of the operations and continue it. In addition, as the driver is influenced by the number of choices, Hick's law that the reaction time is prolonged in proportion to the base 2 logarithm of the number of alternatives is said to be established under simple circumstances. It was reported that large-scale data $(\mathrm{N}=321)$ 
of simple brake reaction maneuvering times by presenting a beat sound from the outside of the vehicle when traveling on a general road in a real car and using a small sample [13]. The result shows that the $25 \%$ of the total number of drivers seem to take at least 1.2 seconds before a braking operation. Another study indicated that the perceived time in the unexpected situation is extended from 0.4 to 1.0 second and the travel time is extended from 0.3 seconds to 0.8 seconds in a test course using an actual vehicle [14]. In most European countries, to avoid accidents when a car travelling in front suddenly stops or decreases in speed, it is advised that the driver of each vehicle must maintain a sufficient distance using either the 2 -second rule or half distance rule. The 2 -second rule is frequently used as a rule of thumb and is taught at driving schools. Some devices (such as road signs) are used to help the driver follow these rules [15].

In this research, the proposed TLS is designed and manufactured using slide rails, mounted on a laboratory racing kart, and a real test run on a circuit is carried out. Kart is used for car driving [16]. Furthermore, a running test on a DS is performed using a handle controller remodeled to a TLS. From the results, the steering operation performance of the conventional steering wheel and the TLS is examined. Furthermore, the steering operation immediately after the cancellation of automatic operation using DS is considered. At that time, we verify the possibility that the driver cannot respond to the sudden change from autonomous control to driver control in an adequate time is verified, thereby creating a dangerous situation.

\section{AUTOMOBILE STEERING MODEL}

It is considered that a human being detects the environment such as the road and the motion condition of a car body and performs the steering operation based on the target exercise situation. The block diagram for this steering operation is shown in Figure 1. A signal is transmitted from the brain to the muscles of the arm, and the steering wheel is then actually operated. Both the biceps brachii and triceps brachii muscles function during the steering operation. These are called bipartite muscles and refer to the muscles straddling the shoulder and the elbow. In addition, a forearm muscle also functions when gripping the steering wheel. Among the forearm muscles, the superficial flexors are greatly involved in the bending motion of the fingers. In this study, we focused on the biceps brachii, which is a bipartite muscle that functions during steering operation and the superficial flexor muscle, which functions when gripping a steering wheel.

Upper limb movement can be considered motion in a two-dimensional plane composed of the shoulder joint (S), elbow joint (E), and carpal joint (W). The motion direction of the upper limb is shown in Figure 2. In the circle whose radius is the distance between the shoulder joint (S) and the carpal joint (W), as in the case of a round steering, the steering operation is performed with vertical direction. For this reason, it is conceivable that a strong grip force is required to maintain a constant steering angle of turn while turning. On the other hand, a TLS performs a steering operation with movement in the normal direction (forward and backward). Therefore, we can maintain the steering angle with weaker grip strength. 


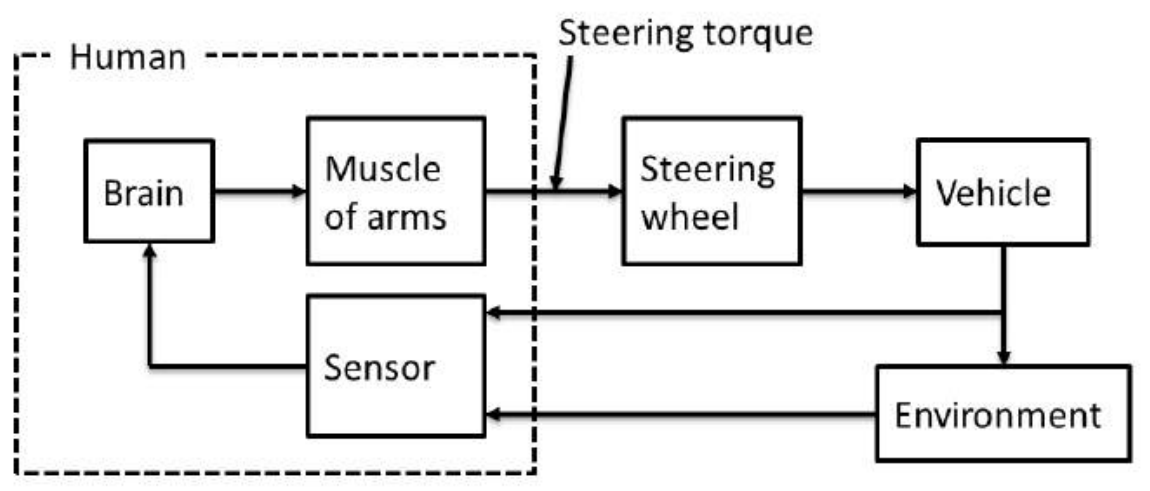

Figure 1. Block diagram for this steering operation.

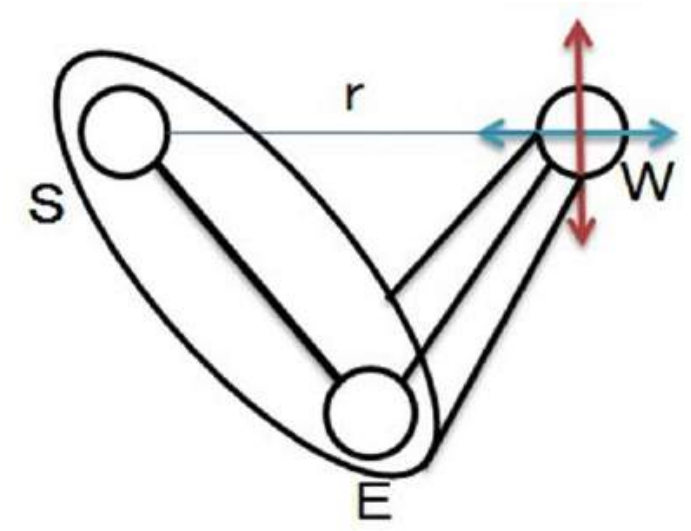

Figure 2. Motion direction of the upper limb.

\section{AUTOMOBILE STEERING MODEL}

The experimental racing kart and the handle controller for DS were also converted to a TLS, enabling operation with a front and rear alternate steering TLS.

\section{Racing Kart Overview}

In this research, Winforce TIA, a Yamaha racing kart, was used as the experimental vehicle. This racing kart has a total length of $1680 \mathrm{~mm}$, a wheelbase of $1040 \mathrm{~mm}$, a maximum full width of $1300 \mathrm{~mm}$, and a dry weight of $58 \mathrm{~kg}$ including the engine. The engine is equipped with Yamaha's KT100SD. The braking system is of the hydraulic disk type and is mounted only on the rear wheels. The Winforce TIA is shown in Figure 3. Table 1 shows the specifications of Winforce TIA and KT100SD. 


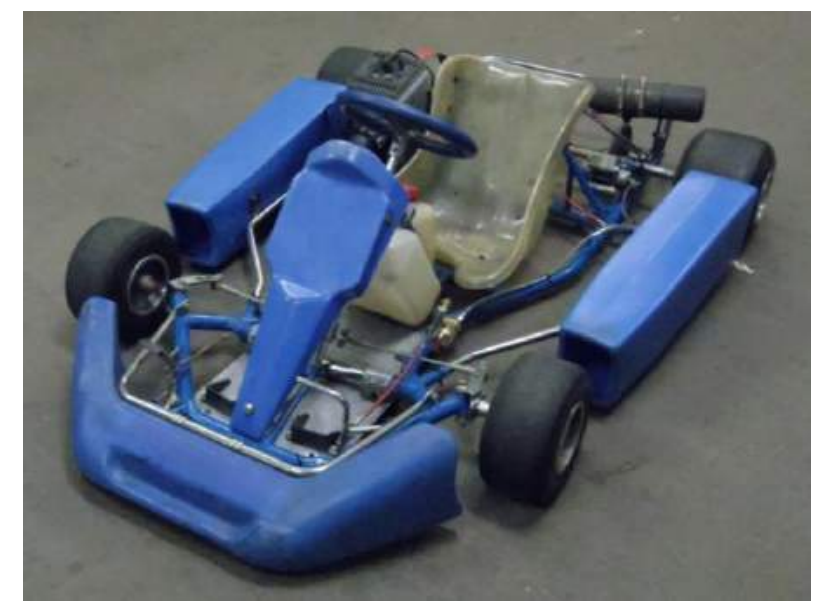

Figure 3. Racing cart: Winforce TIA.

Table 1. Specifications of cart; Winforce.

\begin{tabular}{ll}
\hline Parameters & Specification \\
\hline Total length/Axis Distance & $1680 / 1040 \mathrm{~mm}$ \\
Track F/R & Standard; $1000 / 1300 \mathrm{~mm}$ \\
Dry weight & $58 \mathrm{~kg}$ (including the engine) \\
Toe & $0-1^{\circ}$ \\
Frame Type & Dia.30 mm chrome-molybdenum \\
& steel frame \\
Front tire & $10 \times 3.60-5 \quad$ SL83) \\
Rear tire & $11 \times 6.00-5 \quad$ SL83) \\
Braking system: Rear only & Hydraulic disc \\
Steering system & Directly connected with single stem \\
Engine type & $2-$ stroke, air-cooled, piston valves \\
Cylinder number / Array / Displacement & Single-cylinder / Forward /97.6 cc \\
Bore x stroke & $52.0 \mathrm{x} 46.0 \mathrm{~mm}$ \\
Compression ratio & 7.9 \\
Starting system & Flocked \\
Lubrication system & Mixed gasoline (25: 1) \\
Fuel tank capacity & $8 \ell$ \\
Ignition system & T.C.I. \\
Spark plug & NGK B9EV \\
Carburetor & WALBRO WB-3A \\
Clutch & Direct \\
\hline
\end{tabular}

\section{Design and Production of TLS for Racing Kart}

The TLS was designed and manufactured using a 3590 slide pack FBW made by Linear Guide THK. The stay to which the linear guide is attached consisted of a hollow pipe welded to the frame of the racing kart, and the distance between the rails was determined according to shoulder width. The experimental apparatus consists of Grip part, arm part combined hollow pipe, square pipe. The schematic of the assembly is shown in Figure 4. In addition, in consideration of safety when performing the experiment, a Sparco 6 point seat belt was mounted. The TLS that achieved perfect front and rear alternate steering is 
mounted on a laboratory racing kart as shown in Figure 5 (a), and the view from above is shown in Figure 5 (b).

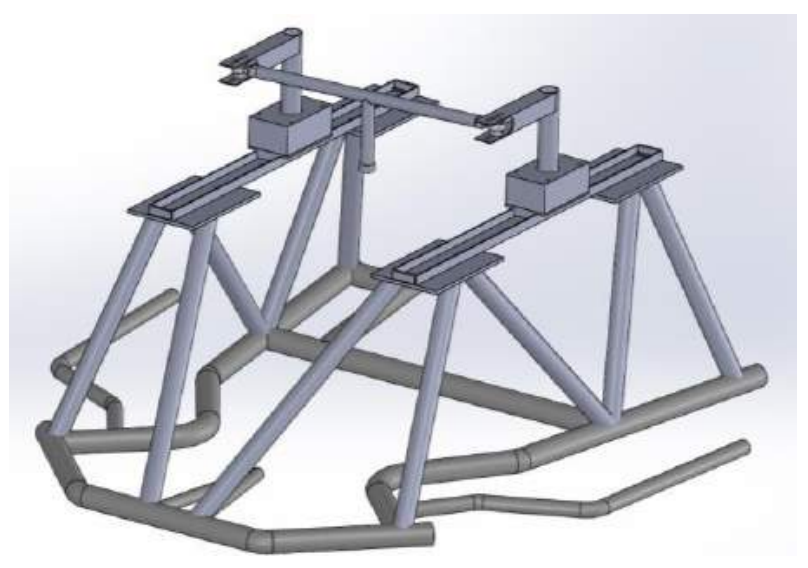

Figure 4. Schematic view of twin lever steering.

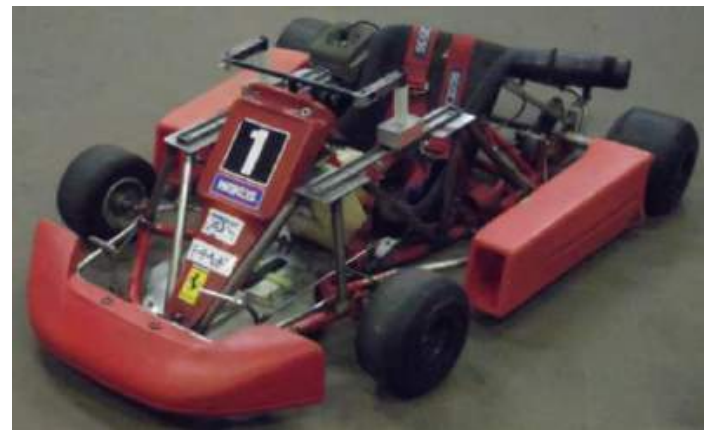

(a)

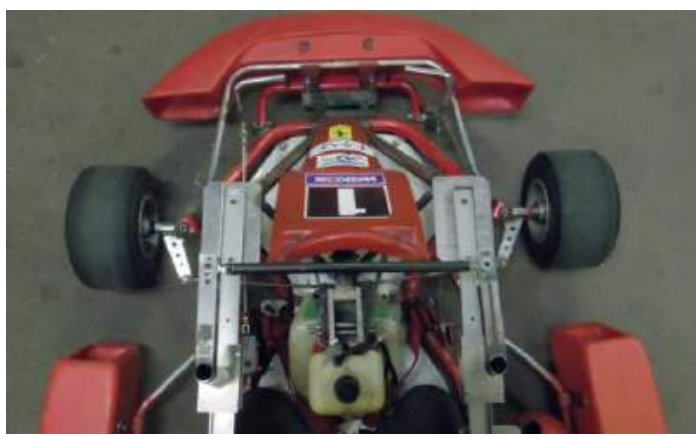

(b)

Figure 5. Racing kart with TLS steering system (a) overview and; (b) steering components.

The round steering of normal racing kart is a 3-spoke steering with an outside diameter of $290 \mathrm{~mm}$. In this racing kart, the Ackermann mechanism is adopted for the steering mechanism, and the mechanism that connects the steering and the steering shaft is both a direct steering type and a round type steering. The proposed TLS achieved the same cutting angle as ordinary steering. In the TLS, the maximum break angle was obtained at around $220 \mathrm{~mm}$ from the neutral point, and in round steering, the maximum break angle was set at $90^{\circ}$. Figure 6 compares the TLS and round steering angle. The steering angle of TLS system is shown in Figure 6 (a) and the steering angle of round type steering system is shown in Figure 6 (b), respectively. The steering angle $\theta$ was set to $30^{\circ}$ for both machines.

\section{TLS Control of Handle Controller}

In order to achieve a complete forward and backward alternate steering TLS in DS, the steering wheel controller was remodeled. The G25 Racing Wheel made by Logitech was used as the handle controller. First, the steering wheel controller was attached upwards and the steering wheel was removed, then the same control as the TLS on the experimental racing kart was installed. The TLS experiment in DS is shown in Figure 7. 


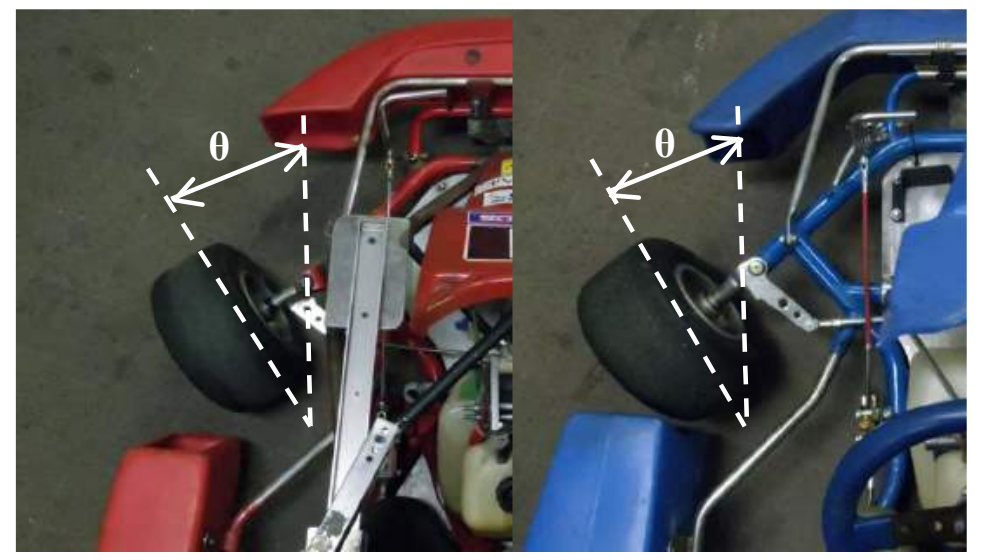

(a)

(b)

Figure 6. Angle of the front wheel $\theta$ (a) TLS system and: (b) round steering.

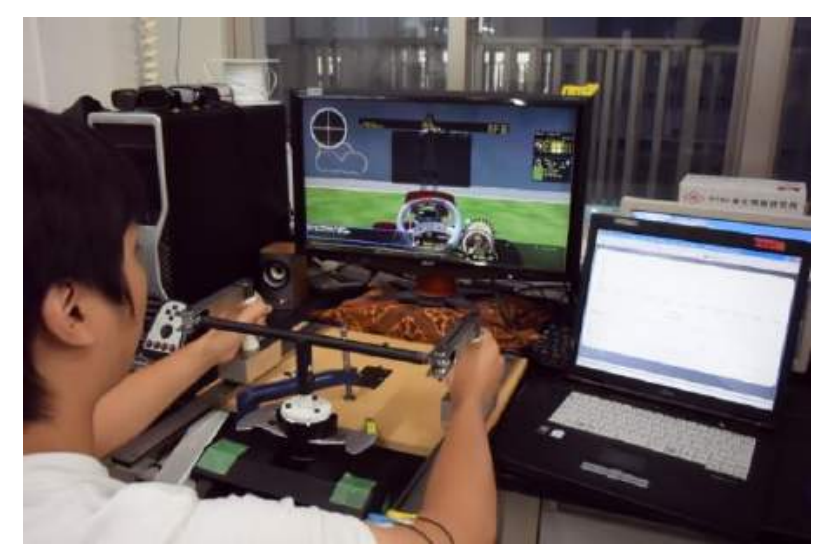

Figure 7. TLS experiment in DS.

\section{AUTOMOBILE STEERING MODEL}

\section{Experimental Method and Results}

In this study, the superiority of TLS would be clarified by running experiments; the relevance of the running experiment to the DS experiment is also examined. First, a real running test is carried out in the experimental racing kart. Myoelectric potential measurement and lap time measurement is performed simultaneously. Next, myopotential measurement and lap time measurement of the subject are also performed in the DS. Myoelectric potential is the voltage that occurs when moving a muscle. How much and how the muscle action potential occurred was investigated.

In this experiment, ProComp 5 Infiniti, an encoder manufactured by Thought Technology Ltd, which is a multi-channel battery-driven device for data collection, was used. This measurement system is shown in Figure 8. A myoelectric electrode was attached with surgical tape. The black electrode is the body earth, and the blue and yellow electrodes are the anode and the cathode. At the time of measurement, noise was removed by wiping the measurement site with alcohol and mounting a disposable electrode on the myoelectric electrode. During measurement, myoelectric electrodes were attached to the biceps brachii muscle that generates the muscle force of the arm and the superflexi flexor 
muscle that generates the grip strength. Measurements were carried out for both the TLS and round steering. The subject was a 22-year-old male and experiments were conducted on a course of 20 laps.

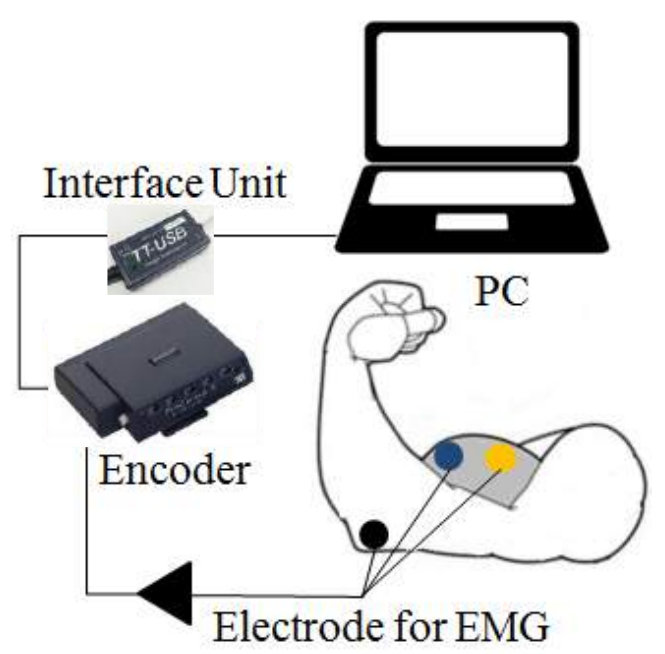

Figure 8. Schematic of EMG measurement system.

\section{Experimental Test Course}

The course shown in Figure 9 was created for the experiment. After passing through the start line, the kart must pass through a large pylon slalom, then a swing corner, a fine pylon slalom, and another swing corner. By installing both a fine pylon slalom and a wide pylon slalom on one course, it is possible to study the steering operation requiring quick fine movement of the arm as well as the reaction of the steering operation when the arm makes large movements. The pylon interval of the fine pylon slalom is $9 \mathrm{~m}$, the pylon interval of the large pylon slalom is obliquely $14 \mathrm{~m}$, and the interval between the $1 \mathrm{st}$ and the $3 \mathrm{rd}$ is set at $20 \mathrm{~m}$; the total lap distance is approximately $200 \mathrm{~m}$. Since the course was asymmetric, myoelectric measurement was performed on both arms. In addition, in the actual run experiments, it was necessary to ensure that the conditions of the experimental vehicles were the same other than the steering method. The experimental vehicle equipped with the TLS and the experimental vehicle equipped with ordinary round steering are equipped with the same engine, even though the vehicle is different. All four tires were the same tires used on each vehicle and the tires to be mounted on the experimental vehicle were unified.

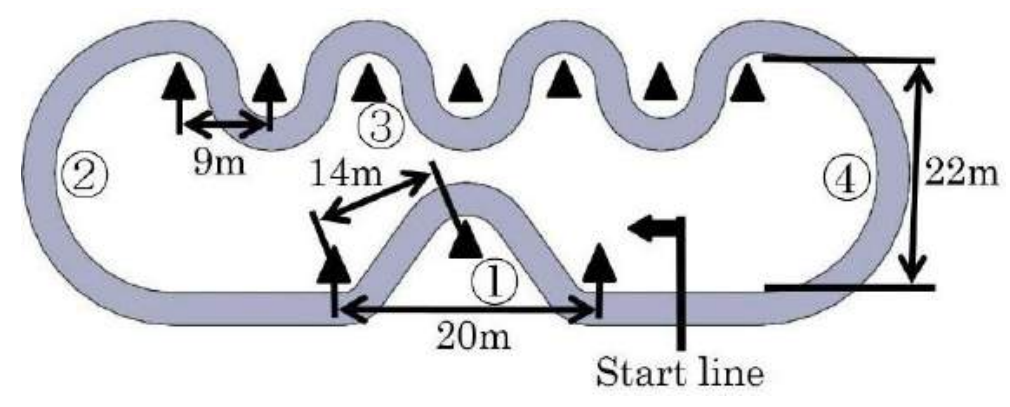

Figure 9. Test course. 


\section{Kart Running Test Results}

The comparison between the lap times for normal round steering and TLS is shown in Figure 10. The average lap time for the entire lap was 0.64 seconds faster for the TLS. In addition, after ten laps, the lap times became faster due to familiarity with the TLS. On the other hand, with the normal round steering, the lap time was slower due to fatigue of the arm. The final lap time was 3.13 seconds faster for the TLS. Figure 11 shows the electromyogram of biceps brachii muscle of the right arm for the last lap of 20-lap experiment. In addition, the electromyogram of the bipod muscle of the right arm for the 20th lap is shown in Figure 12.

Figure 11 (a) shows the results of the normal round steering, and Figure 11 (b) shows the results with the TLS. For smoothing, the data was subjected to moving average processing for 1 second. In Figure 12 and Figure 13, the moving average line is represented by the solid black line, (A)-(C) represents the mark number in Figure 10. For example, in Figure 10, the marked part of (B) is a pylon slalom, which corresponds to (B) in Figure 11 and Figure 12. These figures show that the TLS requires less muscle strength than round steering. In particular, from Figure 11, the average value of the electromyogram during high-speed cornering of (A) and (C) was reduced by about $52 \%$ with the TLS, and a large difference was observed. From Figure 12, it can be seen that the rounded steering shows a significant change in myoelectric potential throughout the lap. On the other hand, the TLS changes little by one turn. By adopting the TLS, it can be seen that the grip strength required during operation is less than for normal round steering. The average value of the myoelectric potential of the whole lap decreased by about $43 \%$ using the TLS, and it was observed that the TLS was operated with about $1 / 2$ of the grip force of round steering.

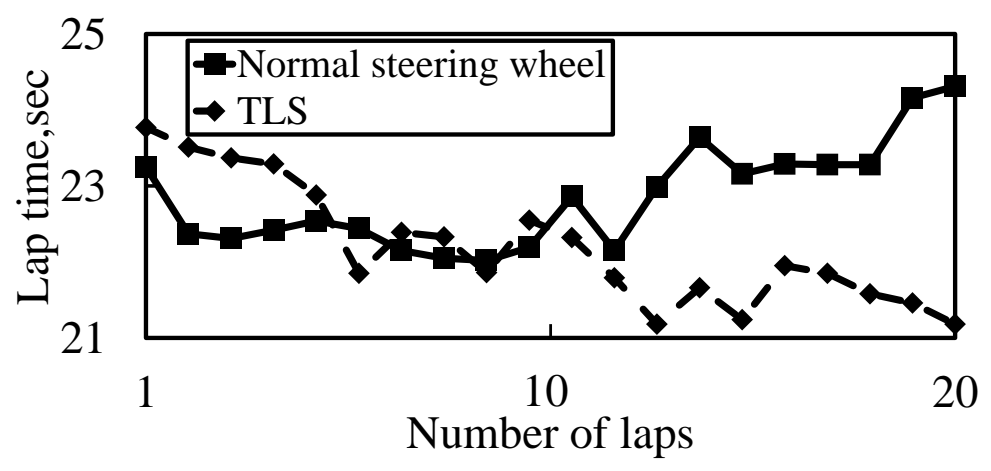

Figure 10. Comparison between the lap times for normal round steering and TLS.

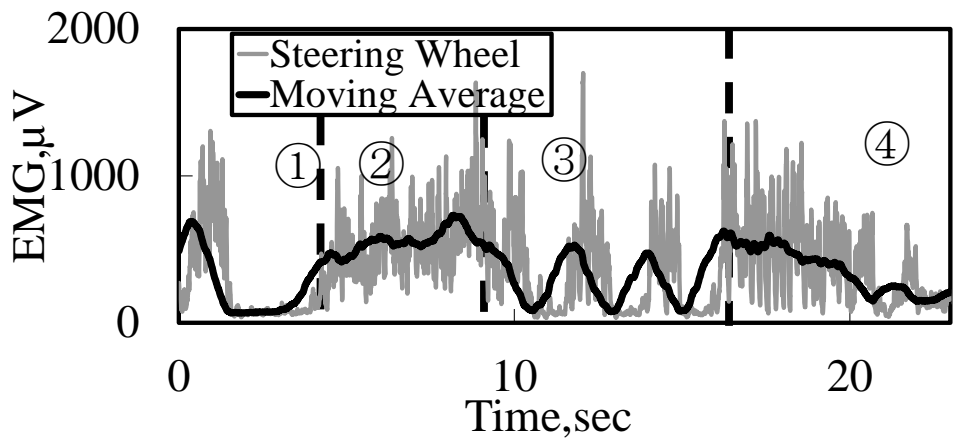

(a) 


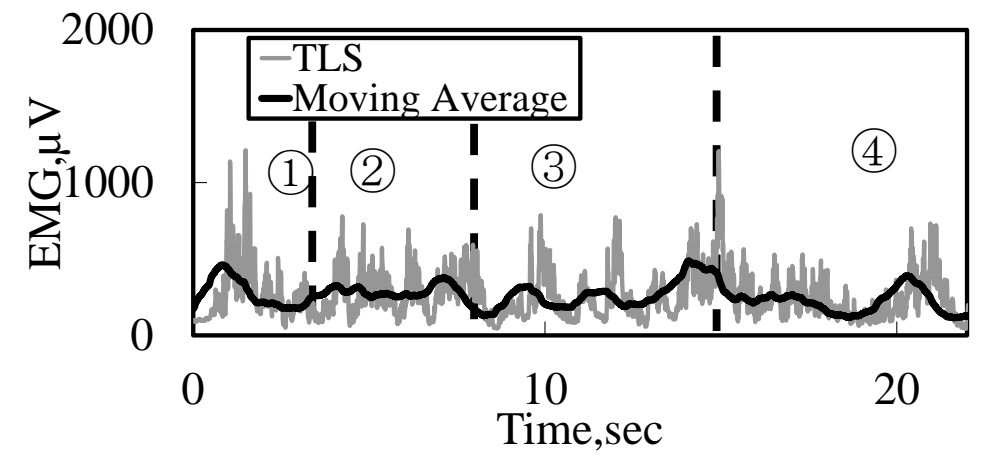

(b)

Figure 11. Electromyogram of biceps brachii muscle of the right arm for the 20th lap of (a) round type steering and; (b) TLS system.

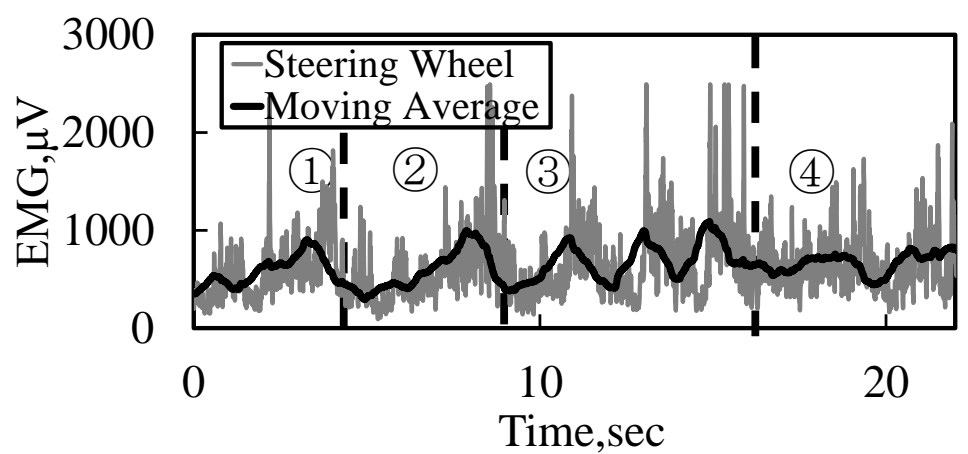

(a)

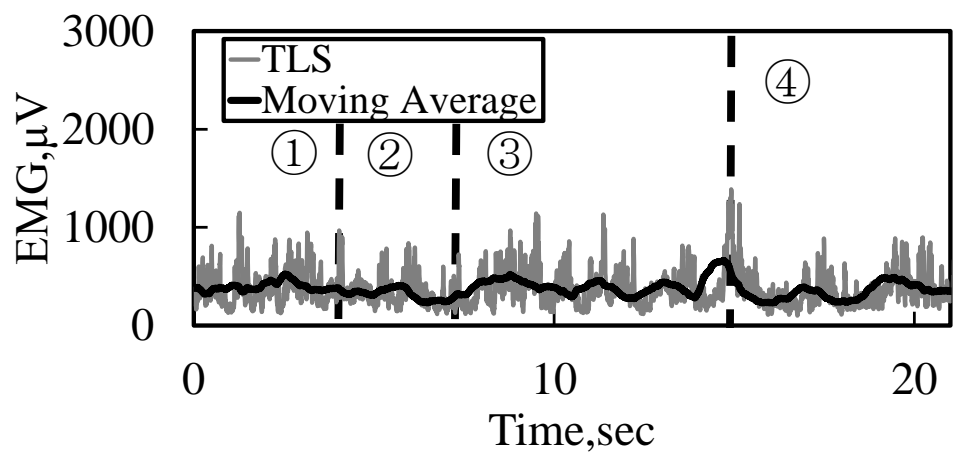

(b)

Figure 12. Electromyogram of flexor digitorum superficialis muscle of the right for the 20th lap of (a) round type steering and; (b) TLS.

\section{DS Experiment Result}

Figure 13 shows the electromyogram of the biceps brachii muscle of the right arm for the last lap of a DS experiment of 20 laps. Figure 13 (a) shows the results with normal round steering, and Figure 13 (b) shows the results with the TLS. From these results, it can be seen that the TLS operates with less muscular strength, similar to the real run experiments. In particular, the average value of the electromyogram during high-speed cornering of (A) to (C) decreased by about $57 \%$ in the TLS experiments, which followed a similar trend to the actual run experiment. 


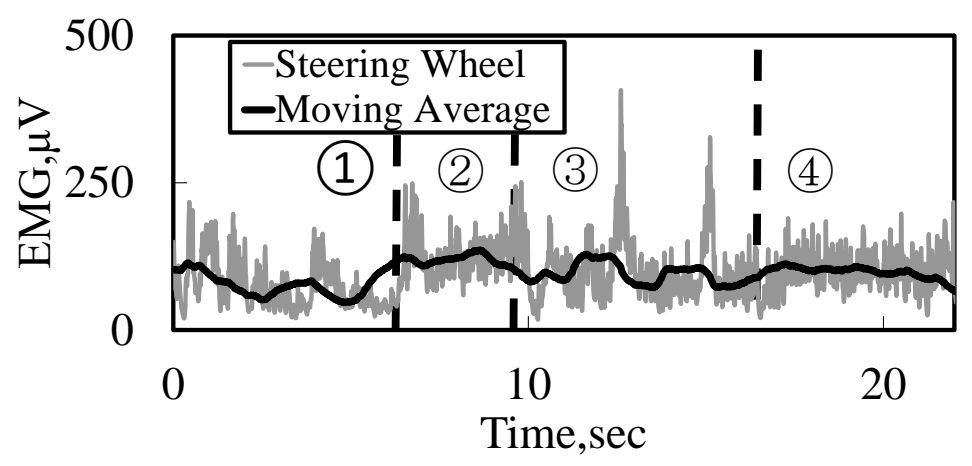

(a)

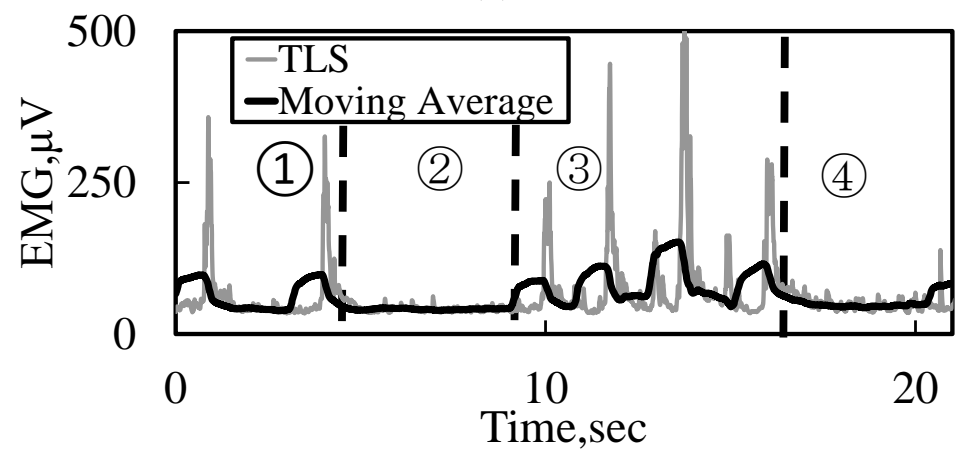

(b)

Figure 13. Electromyogram of biceps brachii muscle of the right arm of DS experiment of (a) round type steering; (b) TLS system.

In addition, the electromyogram of the right arm flexor digitorum superficialis muscle of the last lap of the 20-lap DS experiment is shown in Figure 14. Figure 14 (a) shows the results with normal round steering, and Figure 14 (b) shows the results of the TLS. From these figures, similar to the actual run experiments, the round steering shows that the myoelectric potential changes significantly up and down throughout the lap and that a large muscle force is necessary. On the other hand, the TLS can be operated with less muscular strength. The results of the TLS DS experiments showed the same trend as the actual run experiment that the TLS is operating with less grip strength than the round steering, as the average value of the whole lap decreased by $34 \%$. The results of these electromyogram measurements show the same tendency in both the biceps brachii muscle and the superficial flexor muscle, thus, it was suggested that there is a high correlation between the actual running experiment and the DS experiment.

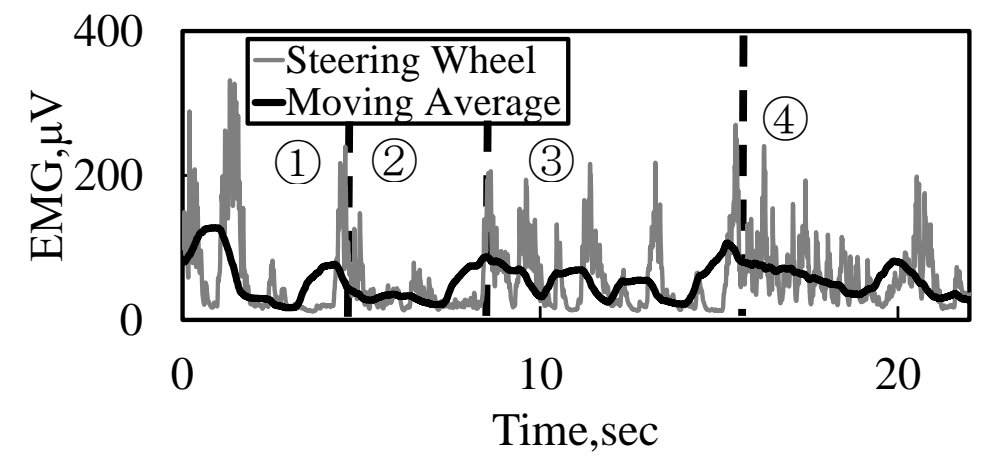

(a) 


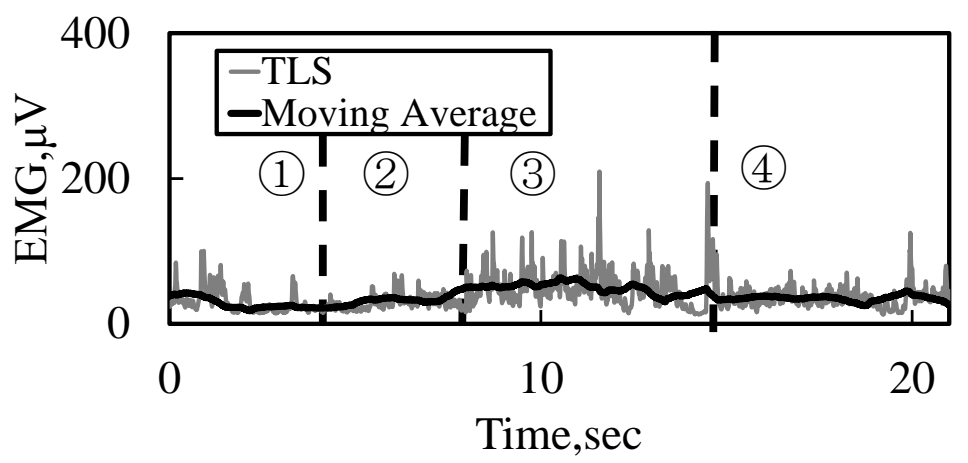

(b)

Figure 14. Electromyogram of flexor digitorum superficialis muscle of the right of DS experiment of (a) round type steering; (b) TLS system.

\section{Comparison of Lap Time and Operation Range}

Next, lap times and distance moved by hand were compared during round steering and TLS operation. The range of motion of the steering wheel was changed by adjusting the cutting angle of the G25 Racing Wheel. Since the round steering wheel has a diameter of $275 \mathrm{~mm}$, the moving distance was calculated from the cutting angle. The average value of five laps was calculated. The lap time and operation range of the round steering were compared with those of the TLS. The relationship between the operating range and lap time is shown in Figure 15. From this figure, it can be seen that there is a difference in the operating range of motion between the TLS and round steering. The fastest lap time for the TLS was in the range of $150 \mathrm{~mm}$ whereas for the round steering the fastest lap time was $350 \mathrm{~mm}$, which was more than twice the difference. For the fastest lap time, the TLS was about $0.3 \mathrm{~s}$ faster. From this result, it was found that the TLS could obtain a fast lap time with less operation range. That is, it is estimated that the control resolution of the arm has improved by adopting the TLS.

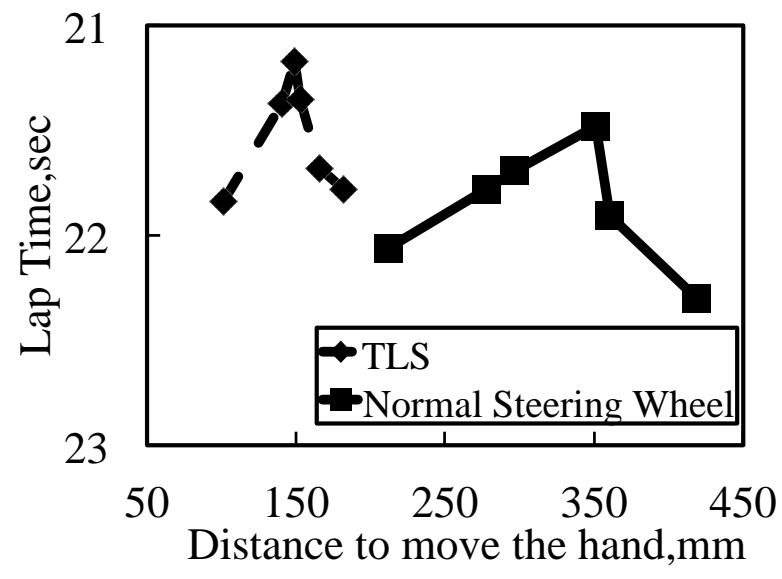

Figure 15. Relationship between the operating range and lap time.

\section{REDUNDANT EXPERIMENT OF AUTONOMOUS CAR OPERATION}

Thus far, real run experiments and DS experiments were conducted, and there was a high correlation between the results. Moreover, the possibility of obtaining high control 
resolution with less muscular strength was indicated by adopting the TLS. The redundancy of automatic driving vehicles was examined by using the result of the experiments. Level 2 automatic driving vehicles are already in practical use, and the practical application of level 3 vehicles is currently being studied. Automatic driving level 3 is a state in which the driver and the automatic driving system coexist to perform the driving operation. In order to achieve level 3 automatic driving, it is necessary to develop a system that safely changes the driving to the driver in an emergency situation where human judgment is necessary. In other words, it is necessary to consider the steering operation performance immediately after the automatic operation is canceled. Therefore, the safety and usefulness of the TLS and normal round steering are examined. At that time, the possibility that the driver cannot respond to the sudden change from autonomous control to driver control in an adequate time is verified, thereby creating a dangerous situation.

\section{Redundancy Experiment Method}

In this experiment, automatic operation was reproduced using the AI (Artificial Intelligence) function of DS. The safety and usefulness of the TLS and normal round steering immediately after the release of automatic operation was examined and the feasibility of Level 3 automatic driving vehicle was considered. It is necessary to determine the delay time when switching from automatic operation to manual operation. Therefore, the delay time was calculated from the stop time of general car. The stop time is calculated as the sum of the reaction time and the brake time. The reaction time is the time from when it is judged that it is necessary for the driver to apply the brake and the brake starts to be effective, and the usual average reaction time is 0.75 second [18]. In addition, the braking time is the time from the start of braking until stopping and can be calculated from Eq. (1).

$t_{b}=\frac{v_{0}}{g}$

where $t_{b}$ is braking time, $v_{0}$ is velocity before braking, is the coefficient friction between the road surface and the tire and $g$ is the gravity acceleleration.

Here, the vehicle speed before braking when traveling on an ordinary road is assumed to be $50 \mathrm{~km} / \mathrm{h}$. The coefficient of friction of the road surface was 0.7 , which is the friction coefficient of dry asphalt. The stop time was calculated and found to be about 3 seconds. Since the stop time while traveling at $50 \mathrm{~km} / \mathrm{h}$ is about $3 \mathrm{~s}$, even when there is redundancy of automatic operation, it is presumed that there is a high possibility that danger can be avoided if the system requests an operation change $3 \mathrm{~s}$ before, therefore a $3 \mathrm{~s}$ grace time was considered in this study.

In the experiment, assuming that the driver fell asleep during autonomous driving, the subject closed their eyes in both the round steering wheel and the TLS experiments, and started the AI running with their hands released from the steering wheel and the lever. The subject was signaled $3 \mathrm{~s}$ before the driving change. The subject judged the information on the screen with a grace period of $3 \mathrm{~s}$ and held the handle or the lever for manual driving. Thease experiments were conducted in randam conditions for emergency avoidance. To make the story simple, these examination were used only control of steering wheel or TLS, but these were not used the brakes. This measurement was performed five times and the number of successful outcomes without spinning was 
counted. In this run experiment, experiments were conducted by five different subjects, the information about each subject (A-E) is shown in Table 2. The gender of the experimental subjects are all males. The number of successful outcomes was also measured when the redundancy grace time was changed from 3 to $2 \mathrm{~s}, 1 \mathrm{~s}$, and no grace time.

Table 2. Experimental subjects.

\begin{tabular}{ccc}
\hline Research subjects & Age & Driving frequency \\
\hline$A$ & 22 & Once a month \\
$B$ & 21 & Once a month \\
$C$ & 22 & Once a week \\
$D$ & 22 & Once a week \\
$E$ & 23 & $2-3$ times a week \\
\hline
\end{tabular}

\section{Result of Redundancy Experiment}

The comparison of the number of successful outcomes of the five subjects in round steering and TLS experiments is shown in Figure 16. From this figure, irrespective of the frequency of driving, the results show that TLS has the same number of successes or many times as TLS in all subjects compared with round steering. In other words, the superiority of the TLS in the steering operation immediately after cancellation of automatic operation is clear. However, even with a grace time of $3 \mathrm{~s}$, the success rate of all subjects did not reach $100 \%$.

It is difficult for the automatic driving system to detect the danger three seconds before and to notify the driver of the change of driving control. Therefore, an experiment was conducted on the same five subjects, A - E, with reduced grace time. The average success count is shown in Figure 17. From this figure, it was found that when the grace time is less than $2 \mathrm{~s}$, the number of successful outcomes is greatly reduced. In particular, when there was no grace time, and the operation was required immediately after cancellation of automatic operation, neither the TLS nor round type steering succeeded.

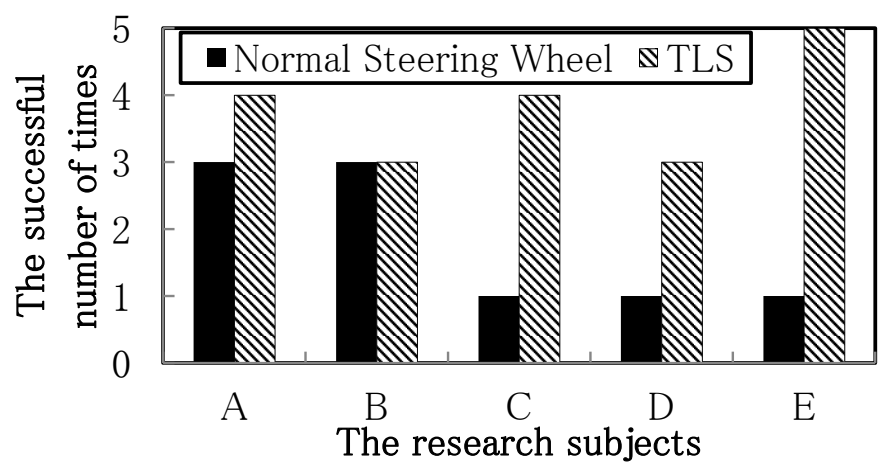

Figure 16. Number of successful outcomes. 


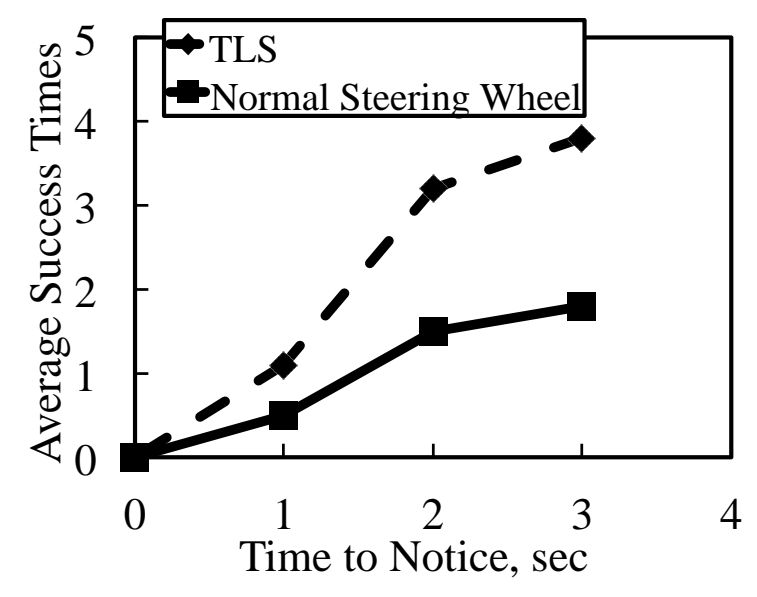

Figure 17. Average number of success times.

\section{CONCLUSION}

We studied the superiority of twin lever steering (TLS) as a new operation method. The following conclusions can be drawn from this study.

a) Compared to conventional round steering, TLS uses less muscular strength, so that arm fatigue is less likely to occur even when the number of laps increases.

b) The myoelectric potential measurement result and the lap time result are similar and related in both real run and DS experiments.

c) In the experiments that considered a steering operation immediately after the cancellation of automatic operation, the TLS had more successful outcomes than conventional round steering and superiority was confirmed when there was a grace time of over $2 \mathrm{~s}$.

d) It is difficult to steer safely when the steering operation is required to occur within 1 second of the cancellation of automatic operation.

e) When there was no time delay ( $0 \mathrm{~s}$ grace time), the number of successes was zero with any of the handles.

In the future, it is considered necessary to optimize the steering performance by changing the steering mechanism to the TLS. Also, since there could be differences in response time from when a stimulus is presented to stepping on brake pedal due to age or gender, it is necessary to collect large scale data that includes gender and age characteristics.

\section{REFERENCES}

[1] Hancock PA, Verwey WB. Fatigue, workload and adaptive driver systems. Accident Analysis \& Prevention. 1997; 29 (4): 495-506. doi : 10.1016/S00014575(97)00029-8.

[2] Zulkarnain N, Zamzuri H, Mazlan SA. Ride and Handling Analysis for an Active Anti-Roll Bar: Case Study on Composite Nonlinear Control Strategy. International Journal of Automotive and Mechanical Engineering. 2014;10:2122242.

[3] Wintera CF, Marieke RH, Martensb H, Stantond NA. Effects of adaptive cruise control and highly automated driving on workload and situation awareness: A 
review of the empirical evidence. Transportation. Research Part F: Traffic Psychology and Behaviour. 2014; 27: 196-217. doi: 10.1016/j.trf.2014.06.016.

[4] Young MS, Stanton NA. Malleable attentional resources theory: A new explanation for the effects of mental underload on performance. Human Factors. 2002; 44: 365-375.

[5] Tajima T, Tada Y, Nakamura Y, Tamura N. Development of the Next-Generation Steering System (Development of the Twin Lever Steering for Production Vehicle) SAE Internationl Journal Passenger Cars - Mechanics System. 2011; 41: 370-383. doi: 10.4271/2011-01-0557.

[6] Pennestri E, Stefanelli R, Valentini PP, Vita L. Virtual musculo-skeletal model for the biomechanical analysis of the upper limb, Journal of Biomechanics, 2007; 40: 1350-1361. doi: 10.1016/j.jbiomech.2006.05.013.

[7] Pick AJ, Cole DJ. Measurement of Driver Steering Torque Using Electromyography. Journal of Dynnamics System and Measurement Control . 2006; 128: 960-968. doi:10.1115/1.2363198.

[8] Mussa-Ivaldi FA, Hogan N, Bizzi E. Neural, Mechanical and Geometric Factors Subserving Arm Posture in Humans. The Journal of Neuroscience, 1985; 10: 2732-2743.

[9] Akamatsu M, Hirasawa S, Hayashi Y. Kansetsu kankaku no kansetstu kakudo•sokudo niyoru chigai nitsuite. Dai 7 kai baiomekanizumu gakujyutukouenkai yokoushu: 1986; 61-64 (in Japanese).

[10] Sakai H. Stability under force control and its index, Transactions of Society of Automotive Engineers of Japan, 2013; 44: 441-448 (in Japanese).

[11] Navarro J, Françoisa M, Mars F. Obstacle avoidance under automated steering: Impact on driving and gaze behaviours. Transportation Research Part F: Traffic Psychology and Behaviour. 2016; 43: 315-324, doi: 10.1016/j.trf.2016.09.007.

[12] Glaser Y, Llaneras R, Glaser D, Green C. Relationship Between Driver Eyes-OffRoad Interval and Hazard Detection Performance Under Automated Driving. SAE Technical Paper. 2016; 2016-01-1424. doi: 10.4271/2016-01-1424.

[13] Johansson G, Rumar K. Drivers' brake reaction time. Human Factors. 1971; 13: 23-27.

[14] Olson PL, Sivak M. Perception-response time to unexpected road way hazards. Human Factors. 1986; 28: 91-96.

[15] Ziedman K. Drivers performance data book. DOT-HS-807-121, National Highway Traffic Safety Administration. Information processing. In R. L. Henderson (Ed.) : 1987; 5.0-5.78.

[16] Abdullah1 NAZ, Sani M.S.M., Husain N.A., Rahman M.M. Zaman I. Dynamics properties of a Go-kart chassis structure and its prediction improvement using model updating approach. International Journal of Automotive and Mechanical Engineering. 2017; 14: 3887-3897. doi: 10.15282/ijame.14.1.2017.6.0316.

[17] Kumamoto M, Oshima T, Yamamoto T. Control properties induced by the existence of antagonistic pairs of bi-articular muscles - Mechanical engineering model analyses. Human Movement Science, 1994; 13: 611-634.

[18] Johansson G, Rumar K. Drivers' brake reaction time. Human Factors. 1971, 13: 23-27. 